\title{
Incidencia detectada del Baculovirus penaei en muestras de post larvas importadas en Costa Rica
}

Ing. Alexander Varela-Mejías

Egresado UNED. Agroindustria. Laboratorio SRY

Departamento de Diagnóstico y Sanidad Acuícola

Sonora, México; alexander.varela@gmail.com

Recibido: 12 de marzo de 2018

Aceptado: 13 de agosto de 2018

\section{RESUMEN}

Se presentan los resultados obtenidos a partir de muestras procesadas en el Laboratorio de Patologías y Parasitología de Crustáceos, entre los años 2012 y 2017. En lo referente al Baculovirus penaei, enfermedad viral que afecta a camarones peneidos principalmente en estadíos larvales y post larvales. Se presentan el número total de muestras recibidas, se tabulan por categoría, por país de origen y por año. Se describe al agente infeccioso, su ruta de infección, las lesiones que genera, su tropismo celular y los efectos que causa en los animales infectados.

Palabras clave: Baculovirus, peneidos, infecciones, post larvas.

\begin{abstract}
Is presented the results obtained from samples processed in the Laboratory of Pathology and Parasitology of Crustaceans, between 2012 and 2017. In relation to the Baculovirus penaei, viral disease that mainly affects penaeid shrimp larvae and post-larvae steps. The total number of received samples are presented, they are tabulated by category, by country of origin and year. The infectious agent, its route of infection, lesions that generates their cell tropism and the effects caused by infected animals is described.
\end{abstract}

Key words: Baculovirus, penaeid, infections, post larvae.

\section{Antecedentes del Baculovirus penaei}

Durante los últimos años, se ha experimentado un incremento en la incidencia, prevalencia e impacto de las enfermedades infecciosas que afectan las producciones acuícolas, incluidas las producciones de camarones marinos. Esto ha dado lugar a grandes pérdidas en aspectos productivos y económicos (Bondad-Reantaso, McGladdery, East y Subasinghe, 2001; Varela y Peña, 2015).

Este fenómeno presenta diversos agentes etiológicos, entre los que destacan múltiples especies de virus, bacterias, hongos y parásitos (Lightner, 1996; Varela, 2016; Varela y Peña, 2017). Se considera además, que el $60 \%$ de las enfermedades que aparecen en la camaronicultura están relacionadas con agentes virales y $20 \%$ a infecciones bacteriales, el resto se distribuye entre diferentes hongos y parásitos (Flegel, 2012).

Entre los agentes virales, el Baculovirus penaei o $\mathrm{BP}$, ha sido reportado como un agente potencialmente causal de mortalidades en los estadíos larvales y post larvales de camarones peneidos, por la Organización Internacional de Epizootias (O.I.E, por sus siglas en francés) y ha sido diagnosticado en cultivos de camarones en Estados Unidos, México, Centroamérica, Colombia, Ecuador, Perú, Brasil y el Caribe (Cuellar-Ánjel, 2015; Morales-Covarrubias y Chávez, 1999; O.I.E, 2016).

El Baculovirus penaei fue renombrado como "Virus de la poliedrosis nuclear con envoltura única del Penaeus vannamei" (PvSNPV por sus siglas en inglés), de acuerdo con el Comité Internacional de Nomenclatura para Virus (Cuellar-Ánjel, 2015), pero se suele seguir denominando como BP en los reportes y artículos. Se le considera un virus de "categoría 2", por su virulencia (Lightner y Pantoja, 2002). 
Entre las especies susceptibles a infecciones por BP, se ha reportado a Penaeus duorarum, $P$. aztecus, $P$. setiferus, $P$. vannamei, $P$. stylirostris y $P$. marginatus. BP también ha sido reportado para $P$. penicillatus, $P$. schmitti, $P$. paulensis y $P$. subtilis (Bondad-Reantaso et al., 2001).

Los signos clínicos del BP no son específicos y carecen de valor diagnóstico, éstos incluyen una reducción en la tasa de crecimiento y alimentación de los organismos infectados, así como un incremento de infestaciones secundarias por epibiontes en la cutícula y branquias de los camarones (Morales-Covarrubias y Chávez, 1999). Anteriormente, se le consideraba un agente causal de serias epizootias, afectado a larvas, post larvas y juveniles de camarones, los animales severamente afectados pueden presentar una coloración pálida en el tracto digestivo (Lightner, 1996).

Los métodos de diagnóstico para el BP incluyen los signos clínicos (diagnóstico presuntivo), el análisis en fresco, la histopatología, uso de sondas moleculares como el Dot blot y la Hibridación in situ; pruebas ELISA, microscopía electrónica, bioensayos y la reacción en cadena de la polimerasa (Cuellar-Ánjel, 2015; Lightner, 1996; Morales-Covarrubias, 2004). De éstas, las más ampliamente utilizadas, por su sensibilidad y accesibilidad, se basan en la observación microscópica de tejidos en fresco o de cortes histopatológicos, mediante la observación de los cuerpos de oclusión piramidales, los cuales se consideran patognomónicos (Cuellar-Ánjel, 2015; Lightner, 1996; MoralesCovarrubias, 2004 y O.I.E, 2016).

Se trata de un virus baciliforme, con envoltura, las dimensiones de su nucleocápside son 286-337nm por 56-79nm. Su genoma está formado por una doble cadena de ADN (BondadReantaso et al., 2001). El tejido blanco son las células epiteliales del hepatopáncreas y se puede encontrar también en el intestino medio (Bondad-Reantaso et al., 2001; Cuellar-Ánjel, 2015; Lightner, 1996; Morales-Covarrubias, 2004, O.I.E, 2016).

Durante las infecciones por BP, las partículas virales pueden estar libres en el nucleoplasma o bien ocluidas en los cuerpos tetraédricos, conformados por una matriz proteína de "poliedrina”. Estos cuerpos de oclusión presentan forma piramidal, pueden ser simples o múltiples, y se acumulan en las células afectadas. Sus dimensiones oscilan entre $0,1 \mu \mathrm{m}$ y $20 \mu \mathrm{m}$ de altura. Por sus dimensiones, los cuerpos de oclusión se pueden observar bajo el microscopio óptico, dentro de los núcleos de células infectadas en muestras de hepatopáncreas, intestino o heces de post larvas o juveniles severamente afectados, y poseen carácter diagnóstico confirmatorio (CuellarÁnjel, 2015; Lightner, 1996; O.I.E, 2016).

En los casos de infecciones leves, las oclusiones son depuradas y no se detectan en animales con pesos mayores a 4 gramos (observación personal). Para su diagnóstico definitivo basta con realizar un análisis en fresco, y se basa en la demostración de la presencia de los cuerpos de oclusión piramidales, intranucleares simples o múltiples en células del hepatopáncreas (Lightner, 1996).

El mecanismo de transmisión utilizado por este patógeno es exclusivamente horizontal, y se da por ingesta de tejido infectado (canibalismo), heces, cuerpos de oclusión, detritos o agua contaminadas con el virus (Bondad-Reantaso et al., 2001; Morales-Covarrubias, $2004 \mathrm{y}$ O.I.E, 2016).

Se han reportado al menos tres cepas geográficas de BP: 1) la de la costa sureste del Atlántico y del Golfo de México de EE.UU. y del Caribe; 2) la cepa de la costa del Pacífico de Sudamérica, Centroamérica y Norteamérica; y 3) la cepa de Hawai (Morales-Covarrubias, 2004 y O.I.E, 2016).

La severidad de la enfermedad será determinada por la susceptibilidad de los animales, por el origen de la cepa presente y por la carga viral, entre otros factores. En eventos de alta virulencia en laboratorios, se producen rupturas celulares de los tejidos afectados, estas células se desprenden y causan atrofia del hepatopáncreas, el cual puede presentar reservas bajas o nulas y pueden desencadenar altas mortalidades. Es poco habitual observar mortalidades elevadas causadas por infecciones de BP en los juveniles y los adultos, pero la infección podría causar un mal crecimiento y una reducción de la 
supervivencia en los estanques de precriadero o de engorde (O.I.E, 2016). Se ha determinado que la infectividad y la patogenicidad del BP, está influenciada por la etapa de desarrollo viral y la edad del hospedador, siendo más virulento durante los estadíos larvales tempranos (Hammer, Stuck y Overstreet, 1998; O.I.E, 2016).

Paralelamente, se ha reportado recientemente que otros patógenos de baja letalidad, pero que al igual que el BP que causan daños en las células del hepatopáncreas, actúan como factores de riesgo para incrementar las mortalidades causadas por infecciones bacteriales secundarias. Debido a que los tejidos lesionados son más fácilmente atacados por bacterias oportunistas (Aranguren, Han y Tang, 2017). Por su parte, Lightner (1996) indica que el desarrollo de brotes de patógenos oportunistas, se incrementa en animales con estado sanitario comprometido.

Es frecuente observar infecciones persistentes en camarones peneidos hospedadores de BP. Las hembras adultas salvajes de $P$. vannamei que están intensamente infectadas por BP excretan heces contaminadas con BP al desovar, y de este modo contaminan los huevos y transmiten el virus a la siguiente generación (O.I.E, 2016).

Para Costa Rica la incidencia del BP en no es reciente, los primeros reportes confirmados de un laboratorio oficializado, datan del año 2008 (datos no mostrados). Pero se carece de antecedentes históricos previos, por lo que no es posible definir si la incidencia está estable o se encuentra cambiante. Es necesario colectar más datos para poder definir el estatus de este patógeno para el país.

Costa Rica, para cubrir las necesidades de post larvas para sus granjas camaroneras, depende enteramente de la importación de animales en estadios larvales o post larvales, ante la ausencia de laboratorios de maduración. Estos lotes de animales son, generalmente monitoreados en su calidad zoosanitaria enfocados en las enfermedades actualmente listadas ante la O.I.E, lista de la cual el Baculovirus penaei ha sido excluido, posibilitando el ingreso de lotes positivos.

Con estos antecedentes, se presentan a continuación los resultados de las muestras recibidas y procesadas por el Laboratorio de Patologías y Parasitología de Crustáceos, entre los años 2012 y 2017. En lo referente a la detección de casos positivos a Baculovirus penaei, para las muestras procesadas mediante análisis en fresco y/o histopatología.

Se debe mencionar que no todos los lotes ingresados al país durante el periodo citado, fueron procesados en este laboratorio. Del mismo modo, los resultados presentados aplican a las muestras procesadas, a su representatividad y a la sensibilidad del método utilizado. De modo que es posible que hayan ingresado otros lotes positivos a este patógeno, sin detectarse.

\section{Materiales y métodos}

Las muestras, originadas de post larvas importadas o de nauplios desarrollados en laboratorios locales, fueron colectadas durante su recepción en las granjas camaroneras. Estas muestras se conservaron con vida para su análisis en fresco, y/o se fijaron para su procesamiento histopatológico según las técnicas descritas por Bell y Lightner (1988), así como Lightner (1996).

Una vez ingresadas, las muestras se registraron con los códigos del laboratorio, en ellos se incluye las iniciales del analista (AV, Alexander Varela), el año de procesamiento (2012 - 2017) y el número consecutivo único de la muestra. Dado que este laboratorio procesa otros tipos de muestras de camarón, los consecutivos de este estudio no son, necesariamente, continuos entre ellos.

Las muestras analizadas en fresco, se procesaron directamente en el Laboratorio de Patologías y Parasitología de Crustáceos, sin tinción o utilizando verde malaquita como tinción de contraste. Por su parte, las muestras analizadas mediante histopatología, se enviaron fijadas al Laboratorio Nacional de Servicios Veterinarios, donde las láminas fueron montadas y devueltas al Laboratorio de Patologías y Parasitología de Crustáceos para su lectura y reporte final.

Las observaciones de las muestras fueron realizadas utilizando microscopios Olympus $\mathrm{CH} 2$ y CX21, con objetivos de 40x. Las 
imágenes se capturaron mediante una cámara digital Canon SX50 HS y se archivaron como evidencia gráfica.

El diagnóstico positivo de las muestras, se realizó según las metodologías descritas por Lightner (1996) y O.I.E (2016). Y consistió en la detección de los cuerpos de oclusión tetraédricos, simples o múltiples, dentro de los núcleos de las células epiteliales del hepatopáncreas, libres en los lumen tubulares o intestinales. Dichas estructuras se consideran patognomónicas, y poseen por tanto, valor diagnóstico confirmatorio (O.I.E, 2016).

Los datos obtenidos se tabularon por año de muestreo, por origen de los animales y estatus sanitario, como positivo o no detectado.

Los resultados de las muestras positivas fueron informados al productor, y reportadas a la autoridad competente, el Sistema Nacional de Salud Animal, a pesar de que el Baculovirus penaei ya no forma parte de la lista de declaración obligatoria ante la O.I.E.

\section{Resultados}

Durante el periodo comprendido entre los años 2012 y 2017, cubiertos por este trabajo, se procesaron un total de 97 muestras de post larvas, correspondientes a laboratorios pertenecientes a cuatro países, Guatemala, Honduras, Ecuador y Nicaragua. De estas, 25 fueron positivas para Baculovirus, representando un $25,7 \%$ del total. Tal como se muestra en la tabla 1.

TABLA 1

Tabulación de resultados para las muestras de post larvas en el periodo 2012 - 2017

\begin{tabular}{ccc} 
Muestra & Resultado & Origen \\
AV-2012-0009 & ND & Guatemala \\
AV-2012-0010 & ND & Guatemala \\
AV-2012-0015 & ND & Guatemala \\
AV-2012-0016 & ND & Guatemala \\
AV-2012-0022 & Positiva & Guatemala \\
AV-2012-0023 & ND & Guatemala \\
AV-2012-0026 & ND & Guatemala \\
\hline
\end{tabular}

TABLA 1 (Continuación)

\begin{tabular}{|c|c|c|}
\hline Muestra & Resultado & Origen \\
\hline AV-2012-0093 & ND & Guatemala \\
\hline AV-2012-0094 & ND & Guatemala \\
\hline AV-2013-0004 & ND & Guatemala \\
\hline AV-2013-0005 & Positiva & Guatemala \\
\hline AV-2013-0006 & Positiva & Guatemala \\
\hline AV-2013-0008 & Positiva & Guatemala \\
\hline AV-2013-0058 & ND & Ecuador \\
\hline AV-2013-0082 & ND & Ecuador \\
\hline AV-2013-0089 & ND & Ecuador \\
\hline AV-2013-0098 & ND & Honduras \\
\hline AV-2013-0103 & ND & Ecuador \\
\hline AV-2013-0122 & ND & Ecuador \\
\hline AV-2013-0138 & ND & Ecuador \\
\hline AV-2014-0001 & ND & Ecuador \\
\hline AV-2014-0011 & ND & Ecuador \\
\hline AV-2014-0646 & ND & Ecuador \\
\hline AV-2014-1186 & ND & Ecuador \\
\hline AV-2014-1284 & ND & Ecuador \\
\hline AV-2014-0843 & ND & Ecuador \\
\hline AV-2014-1108 & ND & Ecuador \\
\hline AV-2015-0022 & ND & Ecuador \\
\hline AV-2015-0023 & ND & Ecuador \\
\hline AV-2015-0024 & ND & Ecuador \\
\hline AV-2015-0039 & ND & Ecuador \\
\hline AV-2015-0682 & ND & Ecuador \\
\hline AV-2015-0683 & ND & Ecuador \\
\hline AV-2015-0722 & ND & Nicaragua \\
\hline AV-2015-0783 & ND & Ecuador \\
\hline AV-2015-0868 & ND & Ecuador \\
\hline AV-2015-0903 & ND & Ecuador \\
\hline AV-2015-0924 & ND & Nicaragua \\
\hline AV-2015-0966 & ND & Ecuador \\
\hline AV-2015-0977 & ND & Ecuador \\
\hline AV-2015-1028 & ND & Ecuador \\
\hline AV-2015-1070 & ND & Nicaragua \\
\hline AV-2015-1071 & ND & Nicaragua \\
\hline AV-2016-0402 & ND & Ecuador \\
\hline AV-2016-0512 & ND & Ecuador \\
\hline AV-2016-0703 & ND & Guatemala \\
\hline AV-2016-0713 & Positiva & Guatemala \\
\hline
\end{tabular}


TABLA 1 (ContinUación)

\begin{tabular}{|c|c|c|}
\hline Muestra & Resultado & Origen \\
\hline AV-2016-0714 & ND & Guatemala \\
\hline AV-2016-1156 & ND & Nicaragua \\
\hline AV-2016-1255 & ND & Guatemala \\
\hline AV-2016-1324 & ND & Guatemala \\
\hline AV-2017-0033 & ND & Nicaragua \\
\hline AV-2017-Q034 & ND & Nicaragua \\
\hline AV-2017-0035 & ND & Nicaragua \\
\hline AV-2017-0036 & ND & Nicaragua \\
\hline AV-2017-0037 & ND & Nicaragua \\
\hline AV-2017-0038 & ND & Nicaragua \\
\hline AV-2017-0039 & ND & Nicaragua \\
\hline AV-2017-0040 & ND & Nicaragua \\
\hline AV-2017-0041 & ND & Nicaragua \\
\hline AV-2017-0042 & ND & Nicaragua \\
\hline AV-2017-0043 & ND & Nicaragua \\
\hline AV-2017-0044 & ND & Nicaragua \\
\hline AV-2017-Q045 & ND & Nicaragua \\
\hline AV-2017-0046 & ND & Nicaragua \\
\hline AV-2017-0051 & ND & Nicaragua \\
\hline AV-2017-0052 & ND & Nicaragua \\
\hline AV-2017-0053 & ND & Nicaragua \\
\hline AV-2017-0054 & Positiva & Nicaragua \\
\hline AV-2017-0055 & ND & Nicaragua \\
\hline AV-2017-0056 & Positiva & Nicaragua \\
\hline AV-2017-0057 & ND & Nicaragua \\
\hline AV-2017-0089 & Positiva & Nicaragua \\
\hline AV-2017-0090 & ND & Nicaragua \\
\hline AV-2017-Q091 & Positiva & Nicaragua \\
\hline AV-2017-0092 & Positiva & Nicaragua \\
\hline AV-2017-0093 & Positiva & Nicaragua \\
\hline AV-2017-0094 & Positiva & Nicaragua \\
\hline AV-2017-0095 & Positiva & Nicaragua \\
\hline AV-2017-0096 & Positiva & Nicaragua \\
\hline AV-2017-0097 & Positiva & Nicaragua \\
\hline AV-2017-0098 & ND & Nicaragua \\
\hline AV-2017-0099 & Positiva & Nicaragua \\
\hline AV-2017-0100 & Positiva & Nicaragua \\
\hline AV-2017-0125 & ND & Guatemala \\
\hline AV-2017-0126 & Positiva & Nicaragua \\
\hline AV-2017-0127 & Positiva & Nicaragua \\
\hline
\end{tabular}

TABLA 1 (Continuación)

\begin{tabular}{ccc} 
Muestra & Resultado & Origen \\
AV-2017-0128 & Positiva & Nicaragua \\
AV-2017-0129 & Positiva & Nicaragua \\
AV-2017-0130 & Positiva & Nicaragua \\
AV-2017-0131 & Positiva & Nicaragua \\
AV-2017-0132 & Positiva & Nicaragua \\
AV-2017-0152 & ND & Guatemala \\
AV-2017-0153 & ND & Guatemala \\
AV-2017-0311 & ND & Guatemala \\
AV-2017-0461 & ND & Guatemala \\
AV-2017-0954 & Positiva & Guatemala \\
\hline
\end{tabular}

Positiva: Positiva a BP; ND: BP No detectado.

Del total de muestras, Guatemala generó un total de 24, de las cuales 6 resultaron positivas, para un $25 \%$, cuatro de ellas durante el año 2013. Las otras muestras positivas son de los años 2012, 2016 y 2017, presentando una muestra positiva para cada uno de estos años.

En el caso de Honduras por su parte, únicamente se recibió una muestra para análisis, en el año 2013, siendo que en ella no se detectó infección por Baculovirus. Esta muestra unitaria da como resultado $100 \%$ de muestras con infección no detectada. Esto sin embargo, no es representativo, y nos expone a un posible error inducido por la Paradoja de Simpson. Requiriendo de más estudios para disponer de más datos sobre este país.

Las muestras prevenientes de laboratorios de Nicaragua, sumaron un total de 45 , de las cuales se diagnosticaron como positivas a Baculovirus 19 , representando un $42,2 \%$ del total de este país. La totalidad de estas muestras positivas se reportaron durante el año 2017, coincidiendo con el año de mayores muestreos tomados para este país.

Ecuador generó 27 muestras, en ninguna de ellas se detectó al Baculovirus, para un $0 \%$ de prevalencia aparente. Dichas muestras se tomaron de lotes de nauplios que ingresaron a Costa Rica para ser entregadas posteriormente como post larvas durante los años 2012, 2013, 2014 y 
2015, cubriendo casi la totalidad del periodo de estudio. (Tabla 2)

TABLA 2

Resumen de resultados por país de importación, durante el periodo 2012 y 2017

\begin{tabular}{lccl}
\multicolumn{1}{l}{ Muestras } & $\mathrm{n}$ & $\%$ & Años de importación \\
Total general & & & \\
Total & 97 & 100 & $2012-2017$ \\
No detectado & 72 & 74,2 & \\
Positivas & 25 & 25,7 & \\
Guatemala & & & \\
Total & 24 & 100 & $2012,2013,2016,2017$ \\
No detectado & 18 & 75,0 & \\
Positivas & 6 & 25,0 & \\
Honduras & & & \\
Total & 1 & 100 & 2013 \\
No detectado & 1 & 100,0 & \\
Positivas & 0 & 0,0 & \\
Nicaragua & & & \\
Total & 45 & 100 & $2015,2016,2017$ \\
No detectado & 26 & 57,8 & \\
Positivas & 19 & 42,2 & \\
Ecuador & & & \\
Total & 27 & 100 & $2013,2014,2015,2016$ \\
No detectado & 27 & 100,0 \\
Positivas & 0 & 0,0 & \\
\hline & & & \\
\hline & & &
\end{tabular}

El año 2017 (Figura 1), fue el de mayor incidencia reportada para Baculovirus penaei, coincidiendo con ser este un año de muy bajas sobrevivencias (datos no mostrados). Estas bajas sobrevivencias estuvieron mayoritariamente relacionadas a problemas bacteriales en hepatopáncreas, generados por Hepatobacter penaei (NHP) y por diferentes cepas de Vibrio.

Sobre ello, se ha reportado que el ataque por patógenos oportunistas es incrementada en animales con estado sanitario comprometido (Lightner, 1996). Análisis de riesgo realizados para otras especies de patógenos, han demostrado que lesiones en hepatopáncreas, incrementan

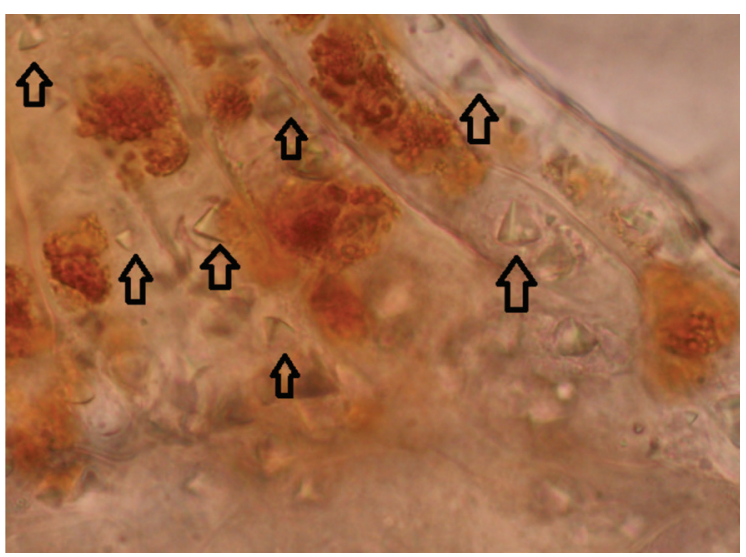

Figura 2. Vista en fresco de hepatopáncreas de post larva fuertemente infectada con Baculovirus penaei, se señalan algunos de los cuerpos de oclusión tetraédricos. 400X, sin tinción.

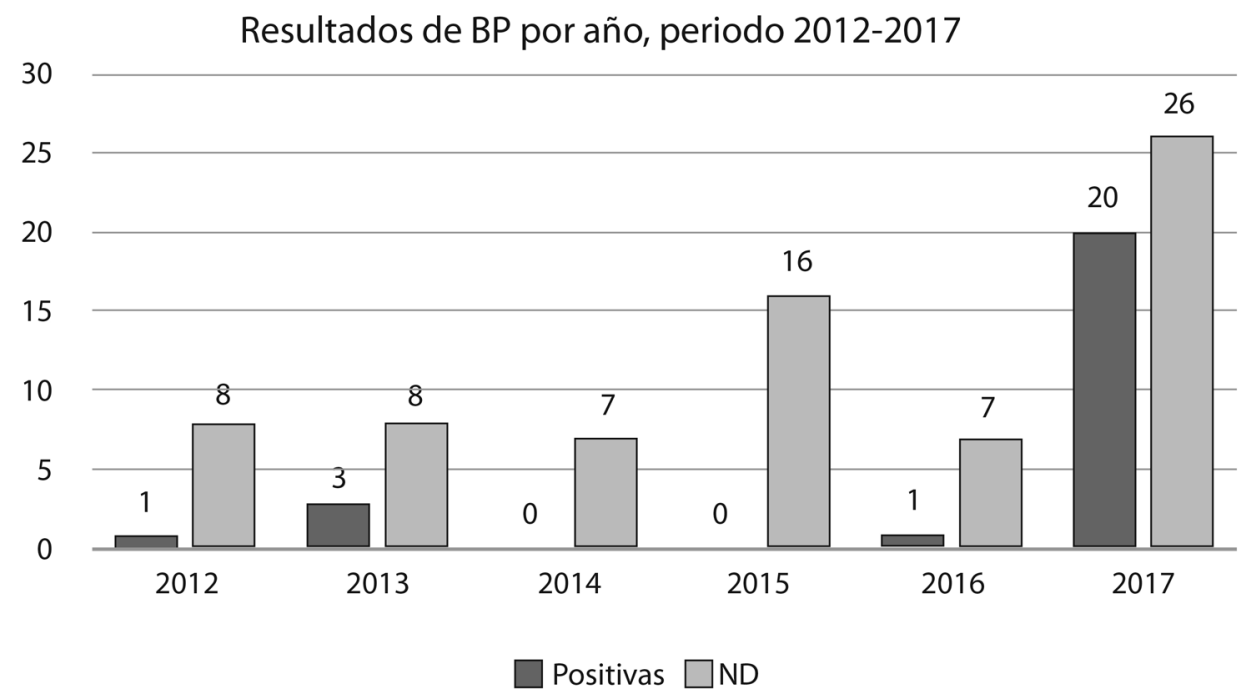

Figura 1. Resultados presentados por año. 
la incidencia de infecciones bacteriales secundarias (Aranguren, Han y Tang, 2017).

No se dispone de investigaciones sobre este efecto para el BP, pero se plantea esta posibilidad, por lo que se requiere de mayores estudios futuros al respecto para confirmar o descartar esta hipótesis.

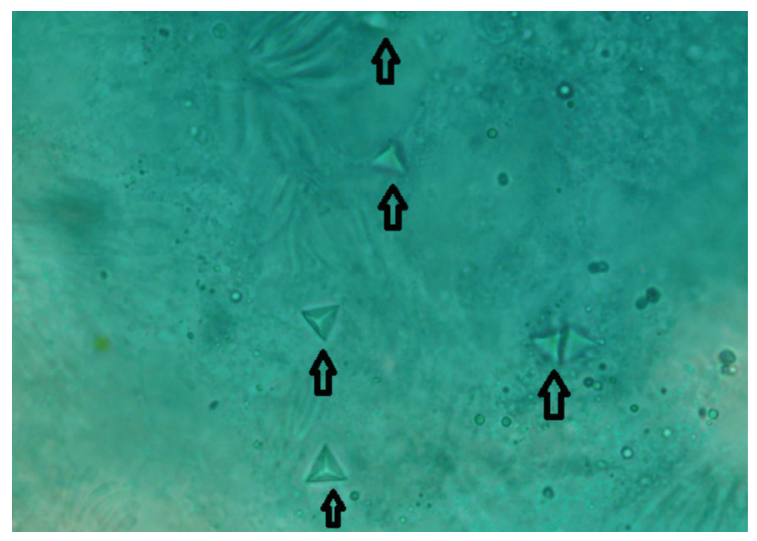

Figura 3. Vista en fresco de impronta de hepatopáncreas de post larva con infección leve por BP, se señalan algunos de los cuerpos de oclusión. 400X, tinción con verde malaquita.

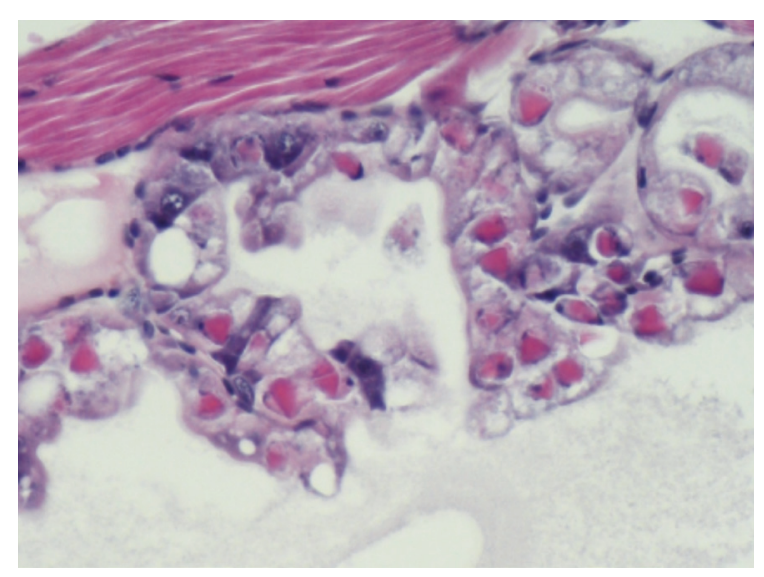

Figura 4. Corte histopatológico de hepatopáncreas de post larva con infección severa por BP, se observan cuerpos de oclusión simples o múltiples, tetraédricos intranucleares y eosinofílicos, en núcleos hipertrofiados con cromatina marginada. 400X, tinción $\mathrm{H} \& \mathrm{E}$.

\section{Consideraciones finales}

Durante los últimos años, infecciones por el Baculovirus penaei se ha detectado y reportado en forma recurrente, para los lotes de post larvas de camarones marinos, importados de diferentes países de origen hacia Costa Rica.

A pesar de ello, no se han realizado estudios sobre la incidencia de estas infecciones y sus posibles efectos en las sobrevivencias finales. Obtenidas en los cultivos. Siendo necesaria la realización de un análisis de riesgos, basado en dichas relaciones. De los resultados de estos estudios, se puede determinar si el BP es un factor de riesgo para infecciones ulteriores o si no existe relación entre BP y las sobrevivencias finales.

Finalmente, y ante la necesidad de importar nauplios o post larvas para sembrar las áreas de cultivo en Costa Rica, se sugiere realizar monitoreos constantes, no solamente para BP, sino para otros agentes potencialmente virulentos, sin importar si forman parte o no, de la lista de declaración obligatoria de la O.I.E.

\section{Referencias bibliográficas}

Aranguren, L, F; Han, J, E \& Tang, K. (2017) Enterocytozoon hepatopenaei (EHP) is a risk factor for acute hepatopancreatic necrosis disease (AHPND) and septic hepatopancreatic necrosis (SHPN) in the Pacific White shrimp Penaeus vannamei. Volume 471, 20 March 2017, p37-42. Aquacuture, 2017.

Bell, T, A; Lightner, D, V. (1988). A Handbook of Normal Penaeid Shrimp Histology. World Aquaculture Society, Baton Rouge, LA, USA.

Bondad-Reantaso, M. G; McGladdery, S. E; East, I. \& Subasinghe. (2001). Asia diagnostic guide to aquatic animal diseases. FAO Fisheries Technical Paper No. 402, Supplement 2. Network of Aquaculture Centres in Asia-Pacific (NACA), and FAO, Rome, ITA.

Cuellar-Ánjel, J. (2015). Baculovirosis tetraédrica. The Center for food security and public health, Iowa State University. Institute for International Cooperation in anima biologics

Flegel T. W. (2012). Historic emergence, impact and current status of shrimp pathogens in Africa. Journal of Invertebrate Pathology 110(2): 174-183.

Hammer, H; Stuck, K, C. \& Overstreet, R, M. (1998). Infectivity and Pathogenicity of Baculovirus penaei (BP) in Cultured Larval and Post larval Pacific White Shrimp, Penaeus vannamei, Related to the Stage of 
Viral Development. Journal of Invertebrate Pathology 72, 38-43 (1998).

Lightner, D. V. (1996). A Handbook of shrimp pathology and diagnostic procedures for diseases of cultured penaeid shrimp. World Aquaculture Society, Louisiana, USA. 304p.

Lightner, D, V \& Pantoja, C. (2002). Bioseguridad en el cultivo de camarones. Organismo di cooperazione e documentazione internazionale. CIDEA. Imprenta Universidad Centroamericana. UCA. Nicaragua.

Morales-Covarrubias, M.S (2004) Enfermedades del camarón: detección mediante análisis en fresco e histopatología. Editorial Trillas, México.

Morales-Covarrubias, M. S \& M. C. Chávez. (1999). Manual para la detección de enfermedades de camarones peneidos utilizando análisis en fresco. Centro de Investigaciones en Alimentación y Desarrollo. México. 68 p.

OIE. (2016). Capítulo 2. 2. 10. Organización Mundial de Sanidad Animal. Manual de diagnóstico en animales acuáticos. $6^{\text {ed. }}$ Paris, Francia.

Varela, A. \& Peña, N. (2015). Hepatopancreatitis necrotizante asociada al Fenómeno del Niño, en cultivos de camarones del Golfo de Nicoya. Repertorio Científico 18(1):29-34.

Varela, A. (2016). Nodavirus de la mortalidad encubierta $(C M N V)$ en camarones marinos de cultivo. Repertorio Científico. Vol. 19, N. ${ }^{\circ}$ 1: Junio 2016: 33-40.

Varela, A. \& Peña, N. (2017). Transfronterización de enfermedades infecciosas en la camaronicultura. Una revisión. Repertorio Científico. Vol. 20, N. ${ }^{\circ}$ 1: Junio 2017: 1-11. 